Recebido em 08/2018. Aceito para publicação em 09/2018.

\title{
DA ATENÇÃO PRIMÁRIA AO SERVIÇO DE REFERÊNCIA NEUROLÓGICA: ANÁLISE DE ENCAMINHAMENTOS E INTEGRAÇÃO ENTRE OS NÍVEIS
}

\section{FROM PRIMARY CARE TO THE NEUROLOGICAL REFERRAL SERVICE: REFERRAL ANALYSIS AND INTEGRATION BETWEEN LEVELS}

\author{
Ronaldo Urias Mendonça ${ }^{1}$ \\ Ronilson Ferreira Freitas ${ }^{2}$ \\ Elaine Veloso Rocha Urias ${ }^{3}$ \\ Antônio Prates Caldeira ${ }^{4}$ \\ Josiane Santos Brant Rocha ${ }^{5}$ \\ Daniela Araújo Veloso Popoff ${ }^{6}$
}

Resumo: Este estudo teve como objetivo verificar a necessidade dos encaminhamentos da atenção primária ao serviço público de referência neurológica, o tempo de espera para esta consulta especializada, a prevalência de patologias atendidas pela neurologia e a integração entre os diferentes níveis de atenção. Tratou-se de estudo epidemiológico, transversal, quantitativo, descritivo que analisou a necessidade, motivo, tempo de espera, concordância entre diagnóstico da atenção primária a saúde e do neurologista, necessidade de exames complementares, destino dos pacientes e integração entre os níveis de assistência. Participaram 400 indivíduos, maioria da zona urbana, femininos, casados, pardos, baixa renda e mais de 5 anos de estudo. O tempo de espera variou de zero a 2357 dias, $68 \%$ dos atendimentos foram primeira consulta, 96,3\% não possuíam ficha de referência e contra referência. Cerca de 50\% dos casos considerados simples não necessitando de acompanhamento neurológico, nem exames complementares. Poucos encaminhamentos possuíam hipótese diagnóstica. As patologias mais prevalentes foram cefaleia, epilepsia e doença cerebrovascular. A análise estatística dos dados utilizando o SPSS® por medidas de tendência central, desvio padrão, frequência simples e relativa, teste qui- quadrado $(p<0,05)$ permitiu concluir que o tempo de espera é longo, não existe integração entre os níveis, nem critérios definidos para encaminhamento.

Palavras-chave: Atenção primária à saúde; neurologia; Saúde Pública.

Abstract: The aim of this study was to verify the need for primary care referrals to the neurological referral public service, the waiting time for this specialized appointment, the prevalence of pathologies treated by neurology and the integration between the different levels of care. This was a descriptive, cross-sectional, quantitative, epidemiological study that analyzed the need, reason, waiting time, agreement between primary health care and neurologist diagnosis, the need for complementary tests, the destination of patients and the integration between care levels. A total of 400 individuals participated in this study, mostly from the

\footnotetext{
${ }^{1}$ Mestre em Cuidado Primário em Saúde pela Universidade Estadual de Montes Claros - Unimontes, Brasil. Email: rum160@outlook.com.br.

2 Doutorando em Ciências da Saúde pela Universidade Estadual de Montes Claros - Unimontes, Brasil. E-mail: ronnypharmacia@gmail.com.

3 Doutora em Ciências da Saúde pela Universidade Estadual de Montes Claros - Unimontes. Brasil. E-mail: urias.elaine@yahoo.com.br.

4 Doutor em Ciências da Saúde pela Universidade Federal de Minas Gerais - UFMG, Brasil. E-mail: antonio.caldeira@unimontes.br.

${ }^{5}$ Doutora em Ciências da Saúde pela Universidade de Brasília - UnB, Brasil. E-mail: josianenat@gmail.com.

6 Doutora em Odontologia pela Universidade Federal de Minas Gerais - UFMG, Brasil. E-mail: danielavelloso@yahoo.com.br.

Revista Univap - revista.univap.br

São José dos Campos-SP-Brasil, v. 25, n. 47, jul. 2019. ISSN 2237-1753
} 
urban area, women, married, brown, with low income and more than 5 years of study. The waiting time varied from zero to 2357 days, $68 \%$ of the cases were first appointment, $96.3 \%$ did not have a reference and counter-reference form. About $50 \%$ of the cases were considered simple, requiring neither neurological follow-up nor complementary tests. Few referrals had a diagnostic hypothesis. The most prevalent pathologies were headache, epilepsy and cerebrovascular disease. The statistical analysis of the data using $S P S S \Theta$ by measures of central tendency, standard deviation, simple and relative frequency, chi-square test $(p<0.05)$ allowed the conclusion that the waiting time is long, there is no integration between the levels nor defined criteria for referral.

Keywords: Primary health care; neurology; Public health.

\section{INTRODUÇÃO}

Em todo o mundo, principalmente, nos países em desenvolvimento, o aumento da expectativa de vida da população tem provocado uma maior prevalência das doenças crônicas não transmissíveis (DCNT), gerando uma demanda crescente aos serviços de saúde, tornando mais acentuados os desafios a serem vencidos pelos gestores públicos (CABRAL, 2013; THEME FILHA et al., 2015). Destacam-se entre estas doenças, patologias neurológicas que afetam um bilhão de pessoas no mundo, com proporção crescente. Epilepsia, doença cerebrovascular, cefaleia, distúrbios dos movimentos, deficit de memória estão entre as patologias neurológicas mais prevalentes e demandam cuidados especiais. A mortalidade por estas condições é de, aproximadamente, 6,8 milhões de pessoas por ano, o que equivale a $12 \%$ das mortes globais, segundo a Organização Mundial de Saúde (OMS) (2004). Esses valores destacam a relevância do problema para os gestores da saúde.

Para o enfrentamento a este e outros problemas decorrentes do novo perfil de morbidade, o Ministério da Saúde no Brasil tem buscado mudar o modelo assistencial, ampliando a cobertura das ações da Atenção Primária à Saúde (APS), visando reduzir as desigualdades assistenciais com ênfase na promoção da saúde e atenção oportuna e precoce à população (BARRETO; CARMO 2007; BERNAL et al., 2016).

A Estratégia de Saúde da Família (ESF) representa o modelo brasileiro de operacionalização da APS. Em tese, as equipes da ESF devem ser habilitadas para resolver a grande maioria dos problemas de saúde da comunidade e atuar na prevenção das doenças, evitando internações desnecessárias e melhorando a qualidade de vida da população (FRACOLLI, 2005). Os casos de maior complexidade devem ser referenciados para atendimento no nível secundário ou terciário. Entretanto, a integração sinérgica entre os diversos níveis de atenção, com ênfase no acesso aos serviços de média complexidade tem sido um grande desafio para o Sistema Único de Saúde (SUS) (LIMA et al., 2016; XIMENES NETO et al., 2009).

Para o atendimento especializado (nível secundário ou terciário) o fluxo do paciente deve ser orientado por formulários específicos de Referência e Contra referência, que são importantes para integrar as informações entre os níveis de atenção e permitir a continuidade do tratamento. Um estudo conduzido no Sul do Brasil revelou 
dificuldade de comunicação entre a APS e a atenção secundária no âmbito das afecções neurológicas, traduzidas em elevado número de encaminhamentos de situações que poderiam ser avaliadas e solucionadas no nível primário (GIOVANELLA, 2006).

Buscando avaliar a integração entre as equipes da ESF e a atenção secundária em neurologia no norte de Minas, onde existe uma reconhecida demanda reprimida de consultas na atenção secundária, o presente estudo teve o objetivo de avaliar os encaminhamentos da atenção primária ao serviço público de referência neurológica, caracterizando a demanda e pertinência/necessidade da avaliação especializada, segundo o profissional do nível secundário.

\section{METODOS}

Trata-se de estudo transversal, exploratório e descritivo, conduzido em Montes Claros, principal polo urbano do norte de Minas Gerais. A cidade contava, na ocasião da coleta de dados, com 72 esquipes da ESF e três centros ambulatoriais de referência para atendimento neurológico. Em pesquisa foi conduzida em um desses centros, um ambulatório vinculado à faculdade de medicina.

Foram considerados elegíveis para o estudo, pacientes maiores de 18 anos, procedentes do próprio município sede do estudo, referenciados por equipes da ESF ao serviço de neurologia ambulatorial. Foram excluídos os pacientes que não possuíam encaminhamento ou quando neste não era possível extrair informações, pacientes que se julgavam incapazes de responder ao questionário, ou cujos acompanhantes avaliassem como tal.

Antes da coleta de dados, conduziu-se um estudo piloto ao longo de um mês para avaliar as estratégias de abordagem aos pacientes, para testar, na prática, o instrumento de coleta e padronizar condutas durante a pesquisa. Em razão da diferença na quantidade de atendimentos/profissional/semana, foi estabelecido um sistema de rodízio entre os três neurologistas (M1, M2, M3) para composição da amostra. Assim, os pacientes que compareceram para a consulta, no período do estudo (novembro de 2013 a novembro de 2014), foram selecionados de forma aleatória e por ordem de chegada. Sendo convidados os primeiros pacientes que preenchessem os critérios de inclusão, até completar 10 pacientes para cada um dos profissionais. Esse procedimento se repetiu ao longo de um ano de coleta de dados, uma vez por semana, para cada profissional.

Os pacientes incluídos na pesquisa, ao responderem ao questionário, informaram procedência, sexo, idade, escolaridade, renda familiar, sinais e sintomas apresentados. A análise dos dados identificou a categoria profissional do responsável pelo encaminhamento (médico, enfermeiro, outros); o tempo de espera para o atendimento neurológico; a existência de ficha de referência e contra referência e a hipótese diagnóstica registrada no encaminhamento. 
Os neurologistas completavam o instrumento de avaliação após realizar consulta, informando sobre a pertinência/necessidade do atendimento, a coerência entre as queixas relatadas pelo paciente e o motivo descrito no encaminhamento, a concordância entre a hipótese diagnóstica do profissional da ESF e do especialista, além de registrarem a solicitação de exames complementares e o destino do paciente. Os três profissionais foram capacitados para o preenchimento da ficha.

Os dados foram submetidos a análises estatísticas pelo programa Statistical Package for the Social Sciences - SPSS $®$ versão 19.0 para Windows. Associação entre as variáveis estudadas e a percepção de pertinência/necessidade, segundo a avaliação do neurologista foram investigadas por meio do teste qui-quadrado ou teste exato de Fisher, considerando o nível de significância de 5\%.

O estudo foi conduzido segundo a Resolução do Conselho Nacional de Saúde (CNS) 466/12, sendo apreciado e aprovado pelo Comitê de Ética em Pesquisa da instituição sede da pesquisa, sob o número 407.480 e todos os participantes assinaram o Termo de Consentimento Livre e Esclarecido.

\section{RESULTADOS}

Ao longo de um ano, foram realizados 1587 atendimentos neurológicos no serviço de referência em que se conduziu o estudo. Nesse período, foram alocados 400 pacientes que preenchiam os critérios de inclusão e concordaram em participar.

O Fluxograma apresentado na Figura 1 discrimina o processo de seleção dos pacientes.

Do total de 400 pacientes envolvidos no estudo, a grande maioria $(94,0 \%)$ era procedente da zona urbana, com idade entre 18 e 88 anos, tendo em média 43 anos (DP=17,49). Registrou-se um discreto predomínio do gênero feminino (54,0\%); 49,8\% dos respondentes informaram ser casados ou viver em união estável. As principais características demográficas e sociais do grupo são apresentadas na Tabela 1, onde se observa ainda que nenhuma variável se mostrou associada à percepção de pertinência/necessidade de encaminhamento, por parte do especialista que fez o atendimento secundário. 
Figura 1 - Fluxograma de seleção dos pacientes para inclusão no estudo.

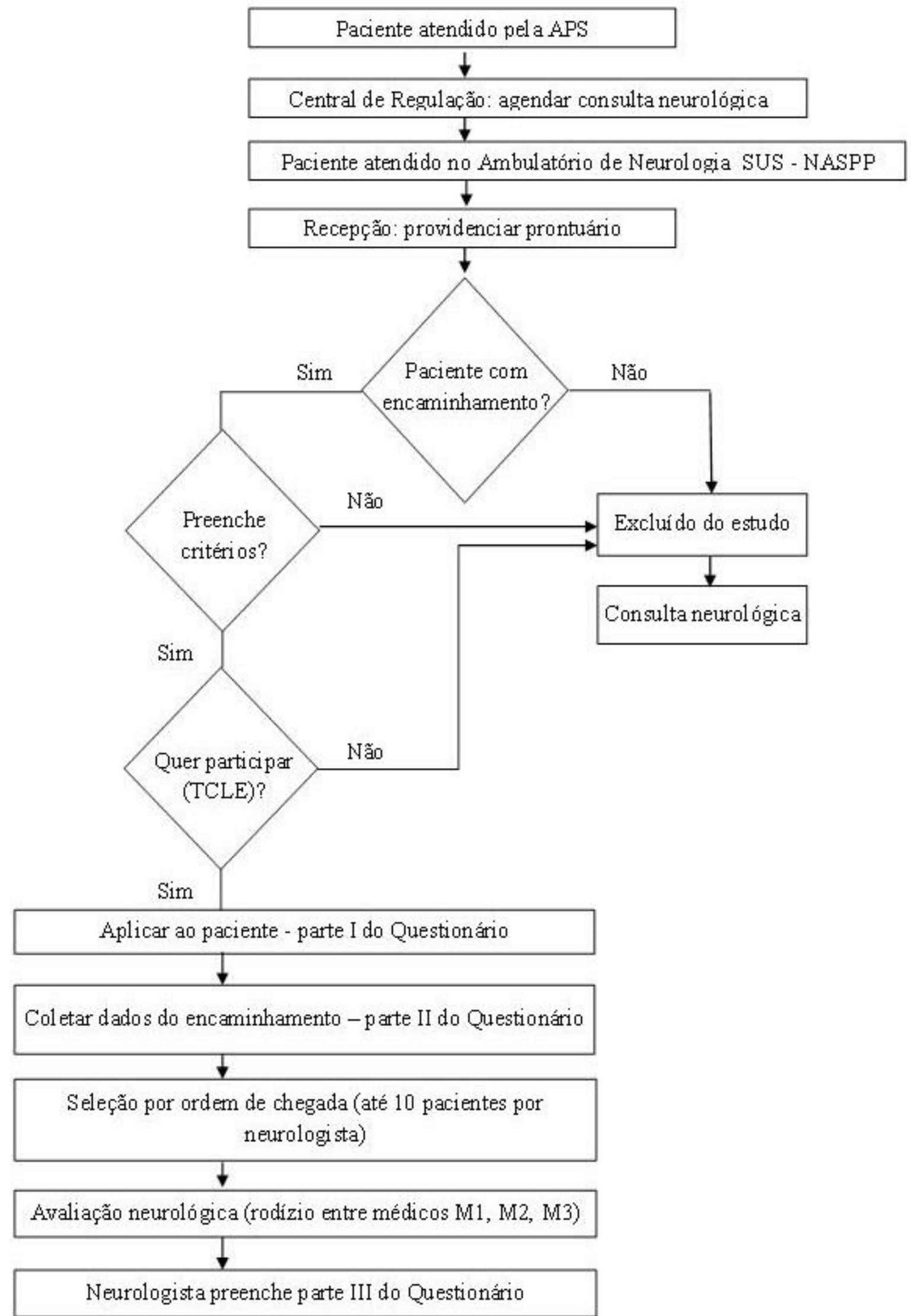


Tabela 1 - Análise comparativa entre grupos de pacientes encaminhados para avaliação neurológica, segundo classificação da pertinência/necessidade de encaminhamento; Montes Claros (MG), 2014.

\begin{tabular}{|c|c|c|c|c|c|}
\hline \multirow[t]{3}{*}{ Variáveis } & \multicolumn{4}{|c|}{ Pertinência/necessidade da consulta neurológica } & \multirow{3}{*}{$\mathrm{p}$-valor* } \\
\hline & \multicolumn{2}{|c|}{ Não } & \multicolumn{2}{|c|}{ Sim } & \\
\hline & (n) & (\%) & (n) & $(\%)$ & \\
\hline Gênero & & & & & 0,869 \\
\hline Masculino & 43 & 45,3 & 141 & 46,2 & \\
\hline Feminino & 52 & 54,7 & 164 & 53,8 & \\
\hline Idade & & & & & 0,721 \\
\hline$<60$ anos & 76 & 80,0 & 249 & 81,6 & \\
\hline$\geq 60$ anos & 19 & 20,0 & 56 & 18,4 & \\
\hline Procedência & & & & & 0,882 \\
\hline Rural & 6 & 6,3 & 18 & 5,9 & \\
\hline Urbana & 89 & 93,7 & 287 & 94,1 & \\
\hline Estado civil & & & & & 0,611 \\
\hline Casado/União estável & 47 & 49,5 & 160 & 52,5 & \\
\hline Solteiro/Separado & 48 & 50,5 & 145 & 47,5 & \\
\hline Cor da Pele & & & & & 0,914 \\
\hline Branca & 22 & 23,2 & 69 & 22,6 & \\
\hline Não Branca & 73 & 76,8 & 236 & 77,4 & \\
\hline Escolaridade & & & & & 0,372 \\
\hline$<$ 5anos & 41 & 43,2 & 116 & 38,0 & \\
\hline$\geq 5$ anos & 54 & 56,8 & 189 & 62,0 & \\
\hline Habitantes/domicílio & & & & & 0,118 \\
\hline$\leq 4$ & 69 & 72,6 & 195 & 63,9 & \\
\hline$>4$ & 26 & 27,4 & 110 & 36,1 & \\
\hline Renda familiar & & & & & 0,112 \\
\hline$<5 \mathrm{SM}$ & 87 & 91,6 & 260 & 85,3 & \\
\hline$\geq 5 \mathrm{SM}$ & 8 & 8,4 & 45 & 14,7 & \\
\hline
\end{tabular}

$\left({ }^{\star}\right)$ Teste qui-quadrado.

O tempo decorrido entre o encaminhamento e o atendimento pelo especialista variou de 0 (zero) a 2357 dias, com mediana de 60,5 dias.

Da amostra 68,0\%, foram encaminhados, para primeiro atendimento, 10,8\% para nova consulta e $21,2 \%$ para retorno com exames. A mediana foi de 60,5 dias para primeira consulta $\left(P_{25}=19 ; P_{75}=125\right) ; 144$ dias para nova consulta $\left(P_{25}=58 ; P_{75}=284\right)$ e 
112 dias para retorno com exames $\left(P_{25}=61,5 ; P_{75}=177,5\right)$.

Com relação às principais queixas de pacientes encaminhados para avaliação neurológica, segundo critério de necessidade de encaminhamento, avaliada pelo neurologista, os dados estão apresentados na tabela 2.

Tabela 2 - Principais queixas e diagnósticos de pacientes encaminhados para avaliação neurológica, segundo critério de necessidade de encaminhamento, avaliada pelo neurologista; Montes Claros (MG), 2014.

\begin{tabular}{|c|c|c|c|c|c|}
\hline \multirow[t]{2}{*}{ Queixas } & \multicolumn{4}{|c|}{ Pertinência/necessidade da consulta } & \multirow[b]{2}{*}{$p$-valor* } \\
\hline & 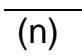 & $(\%)$ & (n) & $(\%)$ & \\
\hline Cefaleia & 38 & 22,5 & 122 & 21,3 & 0,740 \\
\hline Convulsão & 22 & 13,0 & 76 & 13,2 & 0,933 \\
\hline Distúrbios dos Movimentos & 8 & 4,7 & 28 & 4,9 & 0,935 \\
\hline Tonturas & 11 & 6,5 & 31 & 5,4 & 0,587 \\
\hline Transtorno do Humor & 5 & 3,0 & 29 & 5,1 & 0,251 \\
\hline Déficit de Memória & 6 & 3,6 & 19 & 3,3 & 0,882 \\
\hline Trauma Craniano & 6 & 3,6 & 25 & 4,4 & 0,643 \\
\hline Lombalgia & 6 & 3,6 & 17 & 3,0 & 0,701 \\
\hline Déficit de Aprendizado & 4 & 2,4 & 13 & 2,3 & 0,904 \\
\hline Total $^{* *}$ & 169 & 100,0 & 573 & 100,0 & \\
\hline
\end{tabular}

$\left(^{*}\right)$ Teste qui-quadrado ou Teste exato de Fisher

$\left({ }^{* *}\right)$ Alguns pacientes com mais de uma queixa.

Dentre as patologias diagnosticadas pelos neurologistas, as mais prevalentes foram cefaleia $(22 \%)$, epilepsia $(15,5 \%)$, doença cerebrovascular $(8,1 \%)$, traumatismo craniano $(6,0 \%)$, depressão $(5,4 \%)$ (Tabela 3$)$.

Dos entrevistados, apenas 15 pacientes $(3,8 \%)$ foram encaminhados com ficha de referência e contra referência, sendo que $74,8 \%$ foram encaminhados por médicos, $22,5 \%$ por enfermeiros e 2,7 por outros profissionais da saúde, e apenas 99 participantes $(24,7 \%)$ possuíam hipótese diagnóstica no encaminhamento.

Após cada atendimento, os neurologistas completaram o instrumento de avaliação, observando que $76,3 \%$ dos encaminhamentos eram procedentes, em 53,0\% dos casos existia concordância entre as queixas do paciente e o motivo do encaminhamento.

A maioria dos encaminhamentos, 301 (75,3\%) não possuía hipótese diagnóstica, porém, naqueles que possuíam foi verificada concordância entre o médico da APS e o especialista, em $93 \%$ dos diagnósticos, e entre o enfermeiro e o especialista de $33,3 \%$. 
Tabela 3 - Principais diagnósticos de pacientes encaminhados para avaliação neurológica, segundo critério de necessidade de encaminhamento, avaliada pelo neurologista; Montes Claros (MG), 2014.

\begin{tabular}{llllll}
\hline Diagnósticos & \multicolumn{3}{l}{$\begin{array}{l}\text { Pertinência/necessidade } \\
\text { neurológica }\end{array}$} & da & \multicolumn{2}{l}{ consulta } \\
& Não & \multicolumn{5}{l}{ Sim } & \\
\cline { 2 - 5 }$(\mathrm{n})$ & $(\%)$ & $(\mathrm{n})$ & $(\%)$ & p-valor* \\
& 29 & 21,5 & 68 & 14,5 & 0,052 \\
Epilepsia & 11 & 8,1 & 37 & 7,9 & 0,092 \\
Cefaleia Secundária & 9 & 6,7 & 32 & 6,8 & 0,949 \\
Cefaleia Tensional & 5 & 3,7 & 32 & 6,8 & 0,183 \\
Sequela de TCE† & 4 & 3,0 & 31 & 6,6 & 0,110 \\
Sequela de AVE† & 6 & 4,4 & 28 & 6,0 & 0,498 \\
Depressão & 9 & 6,7 & 20 & 4,3 & 0,249 \\
Enxaqueca sem Aura & 4 & 3,0 & 8 & 1,7 & 0,761 \\
Doença de Parkinson & 135 & 100,0 & 469 & 100,0 & \\
Total** & & &
\end{tabular}

$\left({ }^{*}\right)$ Teste qui-quadrado ou Teste exato de Fisher

$\left({ }^{* *}\right)$ Alguns pacientes com mais de um diagnóstico

$(\dagger)$ TCE = Traumatismo Cranioencefálico; AVE = Acidente Vascular Encefálico.

Dos indivíduos avaliados, 53,3\% necessitavam acompanhamento neurológico. Dentre os demais, 6,5\% apresentavam patologias neurológicas simples com alta no primeiro atendimento, $19,0 \%$ poderiam ter tido suas queixas resolvidas pelo médico da APS e $21,2 \%$ deveriam ter sido encaminhados para outros especialistas. Do total de pacientes avaliados, 198 (49,5\%) necessitaram de exames complementares.

\section{DISCUSSÃO}

Este trabalho retratou a situação do atendimento ambulatorial da neurologia a partir do encaminhamento da APS. São escassos os trabalhos na literatura que tratam deste tema e a maior parte do país não conta com estudos na área. $\mathrm{Na}$ análise dos dados, observou-se longo tempo de espera para atendimento com neurologista, tanto para a primeira consulta, como para consultas subsequentes, com registro de pacientes que aguardavam avaliação há mais de seis anos. Em Porto Alegre, estudo realizado em 2009 verificou tempo de espera de até 04 anos (FINKELSZTEJN, et al., 2009). No Canadá, considerado o $10^{\circ}$ país quanto ao tempo de espera para atendimentos especializados, $52 \%$ dos pacientes conseguem atendimento com especialista em 30 dias, sendo a neurologia a $5^{\text {a }}$ especialidade de acesso mais difícil. Na Suíça, é observado o menor tempo de espera, 92\% dos pacientes conseguem consulta com especialista em 30 dias (WAIT TIME ALLIANCE, 2016).

A grande variabilidade do tempo de espera também é um achado relevante. Houve registros de pacientes que foram atendidos no mesmo dia do encaminhamento, 
o que traduz uma falta de coerência no fluxo de pacientes, com priorização de casos, segundo critérios desconhecidos.

A maioria dos pacientes foi encaminhada sem ficha de referência (97\%) e sem hipótese diagnóstica (75,3\%), demonstrando a falta de integração entre os níveis de atenção. Na região da Catalúnia (Espanha), os resultados divergiram desta pesquisa, uma vez que a maioria dos pacientes referenciados da APS aos serviços secundários possuíam ficha de referência e um terço retornavam à APS com ficha de contra referência, o que permite melhor continuidade do tratamento (MARTA-BEATRIZ, et al., 2013).

Em relação ao critério de necessidade de encaminhamento (com ou sem necessidade) avaliado pelos neurologistas, analisando aspectos sócio-demográficos, queixas e diagnósticos não houve diferença estatística significativa entre os dois grupos, o que demonstra a falta de critérios que justifiquem os encaminhamentos ao serviço de neurologia. Estes resultados coincidem com os dados de pesquisa realizada em Huelva, na Espanha (LÓPEZ-DOMíNGUEZ, et al., 2007), que aponta para necessidade de maior seleção dos pacientes a serem referenciados.

Os achados deste trabalho são concordantes com o estudo realizado em Porto Alegre (FINKELSZTEJN, et al., 2009), que concluiu que, apesar de a maioria dos encaminhamentos serem pertinentes, quase metade dos pacientes não necessitava de acompanhamento neurológico, nem de exames complementares, sendo suas patologias consideradas simples, justificando alta no primeiro atendimento neurológico, ou mesmo a condução do atendimento pelo médico da APS ou em outras especialidades.

Estudo multicêntrico realizado na Espanha, concluiu que as taxas de derivações para especialistas eram de $9,0 \%$, sendo $2,5 \%$ do total referenciados à neurologia. Vários fatores interferiam nestas taxas de derivações, como aspectos sócio-demográficos das populações estudadas (idade, prevalência das patologias, população rural ou urbana), do próprio sistema de saúde (oferta de serviços, acessibilidade geográfica, coordenação entre os diferentes níveis, adequação dos protocolos de derivação) e fatores dependentes dos profissionais, como formação, experiência e práticas médicas (SICRAS-MAINAR; NAVARRO-ARTIEDA, 2008). Na presente pesquisa, as taxas de derivações não foram influenciadas pelos aspectos sócio-demográficos, pelas queixas e diagnósticos, o que aponta para a diferença na oferta de serviços e capacitação de profissionais, mas essas variáveis não foram estudadas nesta pesquisa.

Os diagnósticos mais prevalentes, no presente, estudo coincidem com os dados de estudos ambulatoriais realizados em São Paulo (FERRI-BARROS; NITRINI, 1996), Porto Alegre (FINKELSZTEJN, et al., 2009), e nos Estados Unidos (HIRTZ, et al., 2007), e com o estudo de prevalência das doenças neurológicas, realizado pela OMS (WORLD FEDERATION OF NEUROLOGY). Pesquisa realizada em Santa Catarina, com 
pacientes submetidos a perícias médicas, demonstrou resultados discordantes, observando-se elevada prevalência de distúrbios reumatológicos, psiquiátricos, polineuropatias, síndrome pós traumatismo cranioencefálico (TCE), doença cerebrovascular, cefaleia e outras (TREVISOL-BITTENCOURT, et al., 2001). Todavia, é preciso registrar que se trata de um grupo específico de pacientes, uma amostra restrita, tanto do ponto de vista quantitativo, como qualitativo, em que os próprios autores destacam que diversas condições clínicas de natureza não neurológica são encaminhadas para avaliação especializada.

Apesar do crescimento exponencial do número de médicos no Brasil, a distribuição entre as diferentes regiões é muito desigual, com maior concentração nos grandes centros urbanos, a média nacional de profissionais é bem inferior aos países da Europa (SCHEFFER; BIANCARELLI; CASSENOT, 2011), o mesmo ocorre com a neurologia, cujo número é cinco vezes maior na Europa (TREVISOL, et al., 2001).

Para a cidade sede do presente estudo, com uma população residente de, aproximadamente, 400 mil habitantes (IBGE, 2010), e uma população flutuante que atinge mais de um milhão de habitantes, existe uma notável carência de atendimentos por profissionais especialistas na área da neurologia, no âmbito do SUS. Considerando a recomendação do Departamento de Atenção Básica à Saúde do Ministério da Saúde (BRASIL, 2001) quanto ao número de atendimentos ambulatoriais/habitantes/ano, o número de neurologistas em atividade na rede pública e o número de consultas disponibilizadas por mês, conclui-se que os atendimentos não são suficientes para suprir sequer as necessidades do munícipio, criando um longo tempo de espera, o que gera uma grande demanda reprimida e ocasiona risco de agravo à saúde dos pacientes.

Os resultados deste estudo devem ser avaliados à luz de algumas limitações: trata-se de um estudo transversal com amostra que, por restrição logística, não alcançou todos os locais de atenção neurológica do município. Apesar da capacitação dos três neurologistas que preencheram as fichas de coletas de dados, não houve uma avaliação de concordância estatística (kappa) para as avaliações intra ou entre observadores. Contudo, os resultados observados não podem ser negligenciados e devem servir como norteadores para os gestores de saúde da região, ficando constatada a importância de intensificar a comunicação entre os níveis de atenção, elaborar protocolos pertinentes para o atendimento na APS relacionados à área neurológica e sistematizar treinamentos que possam assegurar maior resolutividade no âmbito das equipes da ESF, com vistas em reduzir as demandas de casos que não necessitam de avaliação ou acompanhamento especializado. 


\section{CONCLUSÃO}

Conclui-se que há uma nítida falta de critérios para encaminhamento, tornando o tempo de espera longo e não existe integração entre assistência primária e secundária.

\section{REFERÊNCIAS}

BARRETO, M. L; CARMO, E. H. Padrões de adoecimento e de morte da população brasileira: os renovados desafios para o Sistema Único de Saúde. Ciência \& Saúde Coletiva. v. 12, n.1, p. 1779-90, 2007.

BERNAL, R. T. I. et al. Método de projeção de indicadores das metas do Plano de Ações Estratégicas para o Enfrentamento das Doenças Crônicas não Transmissíveis no Brasil segundo capitais dos estados e Distrito Federal. Epidemiol. Serv. Saude. v. 25, n. 3, p. 455-466, 2016.

BRASIL. Ministério da Saúde. Conselho Nacional de Saúde. Resolução 466/2012. Diretrizes e Normas Regulamentadoras de Pesquisas envolvendo Seres Humanos. Diário Oficial da União oํ 12, 13 Junho 2013 - Seção 1 - Página 59, 2012.

BRASIL. Ministério da Saúde. Secretaria de Políticas de Saúde. Departamento de Atenção Básica (DABS). Parâmetros para programação das ações básicas de Saúde, Brasília: Ministério da Saúde, 2001.

CABRAL N. Epidemiologia e impacto da doença cerebrovascular no Brasil e no mundo. In: NETO. J. P. B.; TAKAYANAGUI, O. M, org. Tratado de Neurologia: Academia Brasileira de Neurologia. São Paulo: Elsevier; 2013. p. 181-189.

FERRI-BARROS, J. E.; NITRINI, R. Que pacientes atende o neurologista. Arquivo de Neuropsiquiatria, v. 54, n. 4, p. 637-44, 1996.

FINKELSZTEJN, A. et al. Encaminhamentos da atenção primária para avaliação neurológica em Porto Alegre, Brasil. Revista de Saúde Coletiva. v. 19, n. 3, p. 731$741,2009$.

FRACOLLI, L. A. O programa de saúde da família como uma estratégia para a inclusão social na perspectiva do trabalho de enfermagem. O Mundo da Saúde, v. 29, n. 1, p. 35-39, 2005.

GIOVANELLA, L. A atenção primária à saúde nos países da União Européia: configurações e reformas organizacionais na década de 1990. Caderno de Saúde Pública. 2006; v. 22, n. 5, p. 951-963.

HIRTZ, D. et al. How commom are the "commom" neurologic disordes. Neurology, v. 68, n. 5, p. 326-37, 2007.

INSTITUTO BRASILEIRO DE GEOGRAFIA E ESTATÍSTICA (IBGE). Censo Demográfico 2010; Infográficos, Montes Claros - MG. Disponível em: $<$ http//www.ibge.gov.br.cidadesat/painel/painel/painel>. Acesso: 23 jul, 2013.

LIMA, E. F. A. et al. Evaluation of the Family Healthcare Strategy from the Perspective 
of Health Professionals. Escola Anna Nery. v. 20, n. 2, p. 275-280, 2016.

LÓPEZ-DOMíNGUEZ, J. M. et al. Análisis descriptivo de la demanda de asistencia neurológica ambulatoria en un área sanitaria de Huelva, Espanã. Revista Neurológica, v. 44, n. 9, p. 527-30, 2007.

MARTA-BEATRIZ, A. et al. Fatores associados a la continuidade assistencial entre atención primaria y atención secundaria ambulatoria experimentada por los usuários en el sistema de salud catalán. Gaceta Sanitária, Espanha, v. 27, p. 207-13, 2013.

ORGANIZAÇÃO MUNDIAL DE SAÚDE (OMS). World Federation of Neurology. Country Resources for Neurological Disorders; Departament of Mental Health and Substance Abuse, Programme for Neurological Diseases and Neuroscience,Geneva; 2004.

SCHEFFER, M.; BIANCARELLI, A.; CASSENOT, A. Org. Demografia médica no Brasil: dados gerais e descrição de desigualdades. São Paulo: Conselho Federal de Medicina, Conselho Regional de Medicina do Estado de São Paulo; São Paulo, 2011.

SICRAS-MAINAR, A.; NAVARRO-ARTIEDA, R. Perfil de eficiência en las derivaciones neurológicas efectuadas a los especialistas de referencia: utilización del sistema de casuística Ajusted Clinical Groups. Anales de Medicina Interna, Madrid, v. 25, n. 5, p. 213-221, 2008.

THEME FILHA, M. M. et al. Prevalência de doenças crônicas não transmissíveis e associação com autoavaliação de saúde: Pesquisa Nacional de Saúde, 2013. Rev Bras Epidemiol. v. 18, n. 2, p. 83-96, 2015.

TREVISOL-BITTENCOURT, P. C. et al. Condições mais frequentes em um ambulatório de neurologia. Arq Neuropsiquiatria, v. 59, p. 214-18, 2001.

WAIT TIME ALLIANCE (WTA), Shedding Light on Canadians' Total Wait for care. Report Card on Wait Times in Canada. june 2012, 1-15. Disponível em: <http://www.waittimealliance.ca/wp-content/uploads/2014/05/2012_Report_Card.pdf>. Acesso em: 13 de out. 2016.

WORLD HEALTH ORGANIZATION (WHO). Atlas - Country Resources for Neurological Disorders: Programme for Neurological Diseases and Neuroscience Departament of Mental Health and Substance Abuse .Geneva, Switzerland: World Federation of Neurology (London), 2004.

XIMENES NETO, F. R. G. et al. Necessidade de qualificação dos enfermeiros da Estratégia de Saúde da Família no Ceará, Brasil. Enfemería Global. v. 17, p. 1-10, 2009. 